\title{
ON THE ASYMPTOTIC BEHAVIOUR OF THE JACKKNIFE FOR STOCHASTIC PROCESSES
}

\author{
P.N. KOKIC AND N.C. WEBER
}

\begin{abstract}
The limiting behaviour of the $J_{\infty}$ jackknife estimator for parameters associated with stochastic processes is shown to depend on the nature of the underlying process through the asymptotic behaviour of the estimator being jackknifed. In particular, the jackknifed versions of certain estimators associated with renewal processes are shown to have an asymptotic normal distribution.
\end{abstract}

\section{Introduction}

Although the original motivation for considering the jackknifing technique was bias reduction, it is the asymptotic properties of the jackknife that make it a useful general tool in data analysis. Gray, Watkins and Adams [3] extended the jackknife technique to estimation problems for certain parameters associated with stochastic processes. They developed the $J_{n}$ estimator by partitioning the observed sample path into $n$ segments and then applying the standard jackknifing procedure to the estimates based on the resulting segments. They proved that if the underlying process was sufficiently well behaved then the bias reduction properties of the jackknife improved as the partition was made finer and this led them to propose the $J_{\infty}$ jackknife estimator.

Gray et al [3] developed the asymptotic theory for the $J_{\infty}$ estimator when the underlying stochastic process has stationary independent

Received 4 January 1983. 
increments. The purpose of this paper is to show that the behaviour of the $J_{\infty}$ estimator depends on the underlying stochastic process through the behaviour of the estimator being jackknifed. This result is then used to obtain some asymptotic results for the $J_{\infty}$ estimator when the underlying process is a renewal process.

\section{Notation}

Let $\{X(t): t \in[a, b]\}$ be a stochastic process and suppose that the probability law of $X(t)$ depends on the parameter $\theta$ for every $t \in[a, b]$. Then if $b \geq t_{2}>t_{1} \geq a$ define $\hat{\theta}\left(t_{1}, t_{2}\right)$ to be an estimator of $\theta$ of the form

$$
\hat{\theta}\left(t_{1}, t_{2}\right)=\left[I\left(t_{2}\right)-I\left(t_{1}\right)\right] /\left(t_{2}-t_{1}\right),
$$

where $\{I(t): t \in[a, b]\}$ is a stochastic process determined by the process $\{X(t): t \in[a, b]\}$, such that almost every realisation of $\{I(t)\}$ is piecewise continuous. The reader is referred to Gray and Schucany [2], Chapter 4, for examples of such estimators. For the purpose of discussing asymptotic results we will take $\{X(t)\}$ defined for all $t \geq a$.

If the process $\{X(t)\}$ is observed for $t \in[a, a+T]$ and we are interested in $f(\theta)$ for some real valued function $f$ then we construct the $J_{n}$ jackknifed estimator for $f(\theta)$ as follows. Let $t_{i}=a+i T / n$, $i=0,1, \ldots, n$ and set $\hat{\theta}=\hat{\theta}(a, a+T)$ and

$$
\hat{\theta}_{n}^{i}=\left[n \hat{\theta}-\hat{\theta}\left(t_{i-1}, t_{i}\right)\right] /(n-1), \quad i=1,2, \ldots, n \text {. }
$$

Define the estimator $J_{n}(f(\hat{\theta}))$ by

$$
J_{n}(f(\hat{\theta}))=n f(\hat{\theta})-(n-1) n^{-1} \sum_{i=1}^{n} f\left(\hat{\theta}_{n}^{i}\right)
$$

By analogy with jackknife estimation results for independent random samples, an estimator for the variance of $J_{n}(f(\hat{\theta}))$ is

$$
s_{n, T}^{2}=\sum_{i=1}^{n}\left[\left[n f(\hat{\theta})-(n-1) f\left(\hat{\theta}_{n}^{i}\right)\right]-J_{n}(f(\hat{\theta}))\right]^{2} /[n(n-1)] .
$$


Another statistic we need to consider is

$$
\hat{\sigma}_{n, T}^{2}=\frac{1}{n([n T]-1)} \sum_{j=1}^{[n T]}\{\hat{\theta}(a+(j-1) / n, a+j / n)-\hat{\theta}(a, a+[n T] / n)\}^{2},
$$

where $[n T]$ denotes the largest integer not exceeding $n T$. If $\operatorname{var} \hat{\theta}(a, a+1)=\sigma^{2}$ and the process $\{I(t)\}$ has stationary independent increments then it is easy to show that $\hat{\sigma}_{n, T}^{2}$ is a consistent estimator for $\sigma^{2}$, as $T \rightarrow \infty$.

Now let $\Gamma$ be the set of possible non-zero jump sizes for the process $\{I(t)\}$. For each $\gamma \in \Gamma$ let $N_{\gamma}$ denote the number of jumps of size $\gamma$ occurring in $I(t)$ for $t \in[a, a+T]$. Set $N=\sum_{\gamma \in \Gamma} N_{\gamma}$. Following Gray et al [3] define the statistics

$$
\begin{aligned}
J_{\infty}(f(\hat{\theta})) & =f(\hat{\theta})-\sum_{\gamma \in \Gamma} N_{\gamma}\left[f(\hat{\theta}-(\gamma / T))-f(\hat{\theta})+(\gamma / T) f^{\prime}(\hat{\theta})\right], \\
s_{T}^{2} & =\sum_{\gamma \in \Gamma} N_{\gamma}(f(\hat{\theta}-(\gamma / T))-f(\hat{\theta}))^{2}
\end{aligned}
$$

and

$$
\hat{\sigma}_{T}^{2}=1 / T \sum_{\gamma \in \Gamma} \gamma^{2} N_{\gamma}
$$

where $f^{\prime}(x)$ denotes the first derivative of $f$ at $x$. Note that if the observed sample path is continuous then $J_{\infty}(f(\hat{\theta}))=f(\hat{\theta})$ and so the $J_{\infty}$ statistic coincides with the original estimator for $f(\theta)$. For a discussion of the bias properties of $J_{n}(f(\hat{\theta})), J_{\infty}(f(\hat{\theta}))$ and the simple estimator $f(\hat{\theta})$ the reader is referred to Gray and Schucany [2].

The following result, proved by Watkins [4], provides sufficient conditions for the convergence of $J_{n}(f(\hat{\theta})), s_{n, T}^{2}$, and $\hat{\sigma}_{n, T}^{2}$ as $n \rightarrow \infty$ and is included for completeness.

THEOREM 1. Let $f$ be a real valued differentiable fronction. Suppose $\{I(t): t \in[a, a+T]\}$ defined above is such that almost every realisation is piecewise continuous and of bownded variation on $[a, a+T]$. 
Further, suppose that for each $t \in[a, a+T], I(t)$ is continuous at $t$ with probability 1 . Then

$$
\begin{aligned}
\lim _{n \rightarrow \infty} J_{n}(f(\hat{\theta})) & =J_{\infty}(f(\hat{\theta})) \text { almost surely, } \\
\lim _{n \rightarrow \infty} s_{n, T}^{2} & =s_{T}^{2} \text { almost surely }
\end{aligned}
$$

and

$$
\lim _{n \rightarrow \infty} \hat{\sigma}_{n, T}^{2}=\hat{\sigma}_{T}^{2} \text { almost sure } z \text {. }
$$

\section{Asymptotic behaviour of the $J_{\infty}$ estimator}

We will now investigate the distribution of $J_{\infty}(f(\hat{\theta}))$ as the record length $T$ becomes larger.

THEOREM 2. Suppose that $\hat{\theta} \stackrel{p}{\longrightarrow} \theta$ and there is a random variable $x$ and a non-negative function $g(T)$ such that

$$
g(T)(\hat{\theta}-\theta) \stackrel{D}{\longrightarrow} X \text { as } T \rightarrow \infty \text {. }
$$

If $f$ is a real valued function with bounded second derivative in a neighbourhood of $\theta, \Gamma$ is a bounded set and $\hat{\sigma}_{T}^{2} g(T) / T \stackrel{p}{\longrightarrow} 0$ as $T \rightarrow \infty$, then

$$
g(T)\left(J_{\infty}(f(\hat{\theta}))-f(\theta)\right) \stackrel{D}{\longrightarrow} f^{\prime}(\theta) X \text { as } T \rightarrow \infty .
$$

Proof. Since $f$ has a continuous derivative in a neighbourhood of $\theta$ it follows that

$$
g(T)(f(\hat{\theta})-f(\theta)) \stackrel{D}{\longrightarrow} f^{\prime}(\theta) X \text { as } T \rightarrow \infty .
$$

So from equation ( 1 ) and Slutsky's Theorem it is sufficient to show that

$$
g(T) \sum_{\gamma \in \Gamma} N_{\gamma}\left[f(\hat{\theta}-(\gamma / T)]-f(\hat{\theta})+(\gamma / T) f^{\prime}(\hat{\theta})\right] \stackrel{p}{\rightarrow} 0 \text { as } T \rightarrow \infty \text {. }
$$

Suppose that $\left|f^{\prime \prime}(t)\right|<M$ for all $t \in(\theta-2 \delta, \theta+2 \delta)$ where $\delta>0$ and let $\gamma_{0}=\sup \{|\gamma|: \gamma \in \Gamma\}$. Then given $\varepsilon>0$, there exists a $T_{\varepsilon}$ such that, for $T>T_{\varepsilon}$, 


$$
\begin{aligned}
& \text { Asymptotic behaviour of the jackknife } \\
& P(\hat{\theta} \in(\theta-\delta, \theta+\delta)) \geq 1-\varepsilon \text { and } \gamma_{0} / T<\delta .
\end{aligned}
$$

Thus, for $T>T_{\varepsilon}$, with probability at least $1-\varepsilon$,

$$
f(\hat{\theta}-(\gamma / T))=f(\hat{\theta})-(\gamma / T) f^{\prime}(\hat{\theta})+\left(\gamma^{2} / 2 T^{2}\right) f^{\prime \prime}\left(\theta_{\gamma}\right),
$$

where $\left|f^{\prime \prime}\left(\theta_{\gamma}\right)\right|<M$. So, for $T>T_{\varepsilon}$,

$$
\begin{aligned}
\left|g(T) \sum_{\gamma \in \Gamma} N_{\gamma}\left[f(\hat{\theta}-(\gamma / T))-f(\hat{\theta})+(\gamma / T) f^{\prime}(\hat{\theta})\right]\right| & =\left|\left(g(T) / 2 T^{2}\right) \sum_{\gamma \in \Gamma} \gamma^{2} N_{\gamma} f^{\prime \prime}\left(\theta_{\gamma}\right)\right| \\
& \leq M \hat{\sigma}_{T}^{2} g(T) / T,
\end{aligned}
$$

with probability greater than $l-\varepsilon$ and the result follows since $\hat{\sigma}_{T}^{2} g(T) / T \stackrel{P}{\longrightarrow} 0$.

Thus the asymptotic behaviour of $J_{\infty}(f(\hat{\theta}))$ depends on the underlying process through the limiting distribution of the estimator $\hat{\theta}$. It is now possible to obtain limit results for $J_{\infty}(f(\hat{\theta}))$ when the underlying process $\{I(t)\}$ does not have stationary independent increments. One immediate consequence of Theorem 2 is the following.

COROLLARY 1. Let $f$ be a real valued fronction with bounded second derivative in a neighbourhood of $\theta$ and suppose $\Gamma$ is a bounded set. If $\hat{\theta} \stackrel{p}{\longrightarrow} \theta$ and $\hat{\sigma}_{T}^{2} / T \stackrel{p}{\longrightarrow} 0$ as $T \rightarrow \infty$ then

$$
J_{\infty}(f(\hat{\theta})) \stackrel{p}{\longrightarrow} f(\theta) \text { as } T \rightarrow \infty .
$$

That is, provided $\hat{\sigma}_{T}^{2} / T \stackrel{p}{\rightarrow} 0$, if $\hat{\theta}$ is a consistent estimator for $\theta$ then $J_{\infty}(f(\hat{\theta}))$ is consistent for $f(\theta)$.

The next result gives a consistent estimator for $f^{\prime}(\theta)$ based on $s_{T}^{2}$

THEOREM 3. Suppose $\hat{\theta} \stackrel{P}{\longrightarrow} \theta$ and $\hat{\sigma}_{T}^{2} \stackrel{p}{\longrightarrow} \eta$ as $T \rightarrow \infty$, for some constant $\eta$. If $f$ is a real valued function with continuous first derivative at $\theta$ and $\Gamma$ is a bounded set then

$$
T_{T}^{2} \stackrel{P}{\rightarrow} n\left(f^{\prime}(\theta)\right)^{2} \text { as } T \rightarrow \infty
$$


Proof. Let $\gamma_{0}=\sup \{|\gamma|: \gamma \in \Gamma\}$ and suppose $f^{\prime}(t)$ is continuous for $t \in\left(\theta-2 \delta_{1}, \theta+2 \delta_{1}\right)$, where $\delta_{1}>0$. So given $\delta_{2}>0$ there is a $\delta_{3}, 0<\delta_{3}<\delta_{1}$, such that

$$
\left|\left(f^{\prime}(t)\right)^{2}-\left(f^{\prime}(\theta)\right)^{2}\right|<\delta_{2} \text { if }|t-\theta|<2 \delta_{3} .
$$

Since $\hat{\theta} \stackrel{p}{\longrightarrow} \theta$, given $\varepsilon>0$ there exists a $T_{\varepsilon}$ such that, for $T>T_{\varepsilon}$,

$$
P\left(|\hat{\theta}-\theta|<\delta_{3}\right)>1-\varepsilon \text { and } \gamma_{0} / T<\delta_{3} .
$$

Hence, for $T>T_{\varepsilon}$,

$$
f(\hat{\theta}-(\gamma / T))=f(\hat{\theta})-(\gamma / T) f^{\prime}\left(\theta_{\gamma}\right)
$$

and

$$
\left|\left(f^{\prime}\left(\theta_{\gamma}\right)\right)^{2}-\left(f^{\prime}(\theta)\right)^{2}\right|<\delta_{2} \text { for all } \gamma \in \Gamma
$$

with probability greater than $1-\varepsilon$. So with probability at least $1-\varepsilon$ we have

$$
\begin{aligned}
\left|T s_{T^{-}}^{2}\left(f^{\prime}(\theta)\right)^{2} \hat{\sigma}_{T}^{2}\right| & =\left|(1 / T) \sum_{\gamma \in \Gamma} \gamma^{2} N_{Y}\left[\left(f^{\prime}\left(\theta_{\gamma}\right)\right)^{2}-\left(f^{\prime}(\theta)\right)^{2}\right]\right| \\
& \leq \hat{\sigma}_{T T_{2}}^{2} .
\end{aligned}
$$

The result now follows since $\delta_{2}$ is arbitrary and $\hat{\sigma}_{T}^{2} \stackrel{P}{\rightarrow} n$ as $T \rightarrow \infty$.

We can now apply the above results to obtain Theorem 6.4 of Gray et al [3] for processes with stationary independent increments.

COROLLARY 2. Suppose $\{I(t): t \geq a\}$ has stationary independent increments, $E \hat{\theta}(a, t)=\theta$ for $t>a$ and $\operatorname{Var} \hat{\theta}(a, a+1)=\sigma^{2}$. If $f$ is a real valued function with bounded second derivative in a neighbourhood of $\theta, \Gamma$ is a bounded set and $\hat{\sigma}_{T}^{2} \stackrel{p}{\longrightarrow} \sigma^{2}$ then

$$
\left\{J_{\infty}(f(\hat{\theta}))-f(\theta)\right) /\left(\left[\sum_{\gamma \in \Gamma} N_{\gamma}(f(\hat{\theta}-(\gamma / T))-f(\hat{\theta}))^{2}\right]^{\frac{3}{2}}\right) \stackrel{D}{\longrightarrow} N(0,1) \text { as } T \rightarrow \infty \text {. }
$$

Proof. Since $\{I(t)\}$ has stationary independent increments, 
$\operatorname{Var} \hat{\theta}(\alpha, a+T)=\sigma^{2} / T$. Writing

$$
\hat{\theta}(a, a+T)=T^{-1} \sum_{i=1}^{[T]} \hat{\theta}(a+i-1, a+i)+\hat{\theta}(a+[T], a+T)(1-[T] / T)
$$

we have from the classical central limit theorem for independent random variables and Slutsky's Theorem that

$$
T^{\frac{1}{2}}(\hat{\theta}-\theta) \stackrel{D}{\longrightarrow} N\left(0, \sigma^{2}\right) \text { as } T \rightarrow \infty \text {. }
$$

Also $\hat{\sigma}_{T}^{2} / T^{\frac{1}{2}} \stackrel{p}{\longrightarrow} 0$ and so the result follows from Theorems 2 and 3 .

In particular, if $\{I(t)\}$ is a Poisson process with intensity $\theta$ then $\Gamma=\{1\}$ and $\hat{\sigma}_{T}^{2}=N_{1} / T \stackrel{p}{\longrightarrow} \theta=\operatorname{Var} \hat{\theta}(a, a+1)$ as $T \rightarrow \infty$. Therefore if $f$ is a real valued function with bounded second derivative in a neighbourhood of $\theta$ then

$$
\frac{J_{\infty}(f(\hat{\theta}))-f(\theta)}{\left[T \hat{\theta}\left(f\left(\hat{\theta}-T^{-1}\right)-f(\hat{\theta})\right]^{2}\right]^{\frac{1}{2}}} \stackrel{D}{\longrightarrow} N(0,1) \text { as } T \rightarrow \infty .
$$

\section{Jackknifing renewal processes}

Suppose we have a stochastic process with events occurring at times

$$
x_{1}, x_{1}+x_{2}, x_{1}+x_{2}+x_{3}, \cdots,
$$

where $\left\{X_{1}, X_{2}, \ldots\right\}$ are independent, non-negative, continuous random variables. Further let $X_{2}, X_{3}, \ldots$ have distribution function $F(x)$ and assume that $E X_{2}=\mu$ and $\operatorname{Var} X_{2}=\sigma^{2}<\infty$. The renewal process $\{I(t): t \geq 0\}$ counts the number of events that have occurred up to time $t$ and is defined by

$$
\begin{aligned}
I(t) & =0 \text { if } x_{1}>t \\
& =\sup \left\{n: \sum_{i=1}^{n} x_{i} \leq t\right\}, \text { otherwise. }
\end{aligned}
$$

In general, $\{I(t): t \geq 0\}$ will not have stationary independent increments. If $X_{1}$ has distribution function $F$ then $\{I(t)\}$ is an ordinary renewal process; if $X_{1}$ has distribution function 
$\mu^{-1} \int_{0}^{x}(1-F(t)) d t$ then $\{I(t)\}$ is an equilibrium renewal process and has stationary increments; otherwise $\{I(t)\}$ is called a modified renewal process.

Consider $\hat{\theta}=\hat{\theta}(0, T)=I(T) / T$, the average number of events per unit time. Since the $X_{i}$ have continuous distributions, the conditions of Theorem 1 are satisfied and so if $f$ is a differentiable, real valued function, $J_{\infty}(f(\hat{\theta}))$ is the almost sure limit of $J_{n}(f(\hat{\theta}))$ as $n \rightarrow \infty$.

Moreover from $\operatorname{cox}[1]$, Section 3.3, we have that

$$
T^{\frac{1}{2}}\left(\hat{\theta}-\mu^{-1}\right) \stackrel{D}{\longrightarrow} N\left(0, \sigma^{2} / \mu^{3}\right) \text { as } T \rightarrow \infty
$$

regardless of the distribution of $X_{1}$, and

$$
\hat{\sigma}_{T}^{2}=T^{-1} \sum_{\gamma \in \Gamma} \gamma^{2} N_{\gamma}=\hat{\theta} \stackrel{p}{\longrightarrow} \mu^{-1} \text { as } T \rightarrow \infty \text {. }
$$

Combining these results with Theorems 2 and 3 we obtain the following theorem for estimates of functions of the mean waiting time for events.

THEOREM 4. Given $\{I(t): t \geq 0\}$ defined above, if $f$ is a real valued function with bounded second derivative in a neighbourhood of $\mu^{-1}$ then

$$
\sqrt{T}\left(J_{\infty}(f(\hat{\theta}))-f\left(\mu^{-1}\right)\right) \stackrel{D}{\longrightarrow} N\left(0,\left[f^{\prime}\left(\mu^{-1}\right)\right]^{2} \sigma^{2} / \mu^{3}\right)
$$

and

$$
T s_{T}^{2}=T^{2} \hat{\theta}\left(f\left(\hat{\theta}-T^{-1}\right)-f(\hat{\theta})\right)^{2} \stackrel{p}{\rightarrow} \mu^{-1}\left[f^{\prime}\left(\mu^{-1}\right)\right]^{2} \text { as } T \rightarrow \infty .
$$

In particular if the coefficient of variation of the waiting time distribution $\sigma / \mu=k$, where $k$ is known, then

$$
\frac{J_{\infty}(f(\hat{\theta}))-f\left(\mu^{-1}\right)}{\left[T \hat{\theta}\left(f\left(\hat{\theta}-T^{-1}\right)-f(\hat{\theta})\right)^{2}\right]^{\frac{3}{2}}} \stackrel{D}{\longrightarrow} N\left(0, k^{2}\right) \text { as } T \rightarrow \infty .
$$




\section{References}

[1] D.R. Cox, Renewal theory (Methuen, London, 1970).

[2] H.L. Gray and W.R. Schucany, The generalized jackknife statistic (Statistics Textbooks and Monographs, 1. Marcel Dekker, New York, 1972).

[3] H.L. Gray, T.A. Watkins and J.E. Adams, "On the jackknife statistic, its extensions, and its relation to $e_{n}$-transformations", Ann. Math. Statist. 43 (1972), 1-30.

[4] T.A. Watkins, "Jackknifing stochastic processes" (PhD dissertation, Texas Technical University, Texas, 1971).

Department of Mathematical Statistics, University of Sydney,

Syoney,

New South Wales 2006, Australia. 